\title{
COMPLICACIONES Y FACTORES DE RIESGO EN PACIENTES LLEVADOS A COLANGIOPANCREATOGRAFÍA RETRÓGRADA ENDOSCÓPICA EN EL HOSPITAL MILITAR CENTRAL EN EL PERIODO DE ENERO DE 2011 A JUNIO DE 2012
}

\author{
Imbeth A. Pedro 1 , Guzman L Isaac', Julliao Haroldo ${ }^{3}$, Salej Jorge ${ }^{3}$, Galvan Camilo \\ ${ }^{1}$ Residente del Programa de Gastroenterología de la Universidad Militar Nueva Granada, \\ Bogotá, D.C., Colombia. \\ ${ }^{2}$ Residente del Programa de Medicina Interna de la Universidad Militar Nueva Granada, \\ Bogotá, D.C., Colombia. \\ ${ }^{3}$ Especialistas Gastroenterólogos, Departamento de Gastroenterología de Hospital Militar Central, \\ Bogotá, D.C., Colombia. \\ ${ }^{4}$ Estudiante de Séptimo Semestre de Medicina. Universidad Militar Nueva Granada, \\ Bogotá, D.C., Colombia. \\ Correspondencia:drplia@yahoo.com \\ Recibido: Octubre 11 de 2013 Aceptado: Enero 22 de 2014
}

\begin{abstract}
Resumen
Objetivos: Describir las complicaciones y los factores de riesgo que se presentaron en pacientes llevados a colangiopancreatografía retrógrada endoscópica (CPRE) en el Hospital Militar Central en el periodo de Enero de 2011 a Junio de 2012.
\end{abstract}

Métodos: Estudio descriptivo retrospectivo. Revisión de historias clínicas. Se describió una cohorte de 96 pacientes llevados a CPRE en el Hospital Militar, haciendo énfasis en las características de los mismos, indicación de CPRE, y complicaciones presentadas posterior a dicho procedimiento. Se realizó una revisión de las historia clínicas de los pacientes que cumplieron con los criterios de inclusión, a las cuales se le aplicó una encuesta con el objetivo de identificar que complicaciones se presentaron en el periodo de tiempo escogido y cuales factores de riesgo podrían estar implicados. Con la prueba Chi cuadrado se evaluó la asociación entre los factores de riesgo y las complicaciones.

Resultados: Se encontró que el $20.8 \%$ de los paciente sometidos a CPRE presento una complicación, siendo las más frecuente pancreatitis aguda con un $11.5 \%$, seguida por infección y sangrado digestivo. La mortalidad fue de $2.1 \%$. La mayoría de las CPRE tenían una indicación excelente y en su totalidad fueron realizadas por personal idóneo desde el punto de vista técnico. No hubo asociación estadística entre los factores de riesgo relacionados con el paciente o la técnica.

Conclusión: Las complicaciones encontradas en nuestros pacientes corresponden con las descritas en la literatura tanto en tipo como en frecuencia. No se encontró ninguna asociación estadísticamente significativa entre la ocurrencia de complicaciones y aquellos factores de riesgo relacionados con el paciente o la técnica.

Palabras claves: Colangiopancreatografía retrograda endoscópica, complicaciones, pancreatitis, perforación intestinal. 


\title{
COMPLICATIONS AND RISK FACTORS IN PATIENTS UNDERGOING ENDOSCOPIC RETROGRADE CHOLANGIOPANCREATOGRAPHY AT HOSPITAL MILITAR CENTRAL IN BOGOTA IN THE PERIOD JANUARY 2011 TO JUNE 2012
}

\begin{abstract}
Objectives: Describe the complications and risk factors within the patients undergoing Endoscopic Retrograde Cholangiopancreatography (ERC) at Military Central Hospital in Bogotá between January 2011 and June 2012.

Methods: Retrospective Descriptive Study. Review of Medical Records. This paper describes a cohort of 96 patients that went through Endoscopic Retrograde Cholangiopancreatography (ERC) at the Military Central Hospital, highlighting their characteristics, ERC indications, and the complications that rose after the procedure. A review of the medical records of the patients that met the inclusion criteria was made to apply a survey in order to identify the complications that arose in the time period chosen, as well as the risk factors that could be involved. With the Chi-square distribution test, the association among the risk factors and the complications were evaluated.
\end{abstract}

Results: It was found that $20,8 \%$ of the patients undergoing ERC had a complication, from which pancreatitis was the most frequent with $11,5 \%$, followed by infection and digestive bleeding. Mortality rate was 2,1\%. Most of the ERC had an excellent indication and were all performed by qualified staff from the technical perspective. There was no statistical association among the risk factors related to the patient or the technique.

Conclusion: The complications that were found in the patients correspond to those described in the literature both in type and frequency. There was no relevant statistical association between the appearances of the complications and those risk factors related to the patient or the technique.

Keywords: Endoscopic Colangiopancreatography Retrograde, complications, pancreatitis, intestinal perforation.

\section{COMPLICAÇÕES E FATORES DE RISCO EM PACIENTES COM COLANGIOPANCREATOGRAFIA RETRÓGRADA ENDOSCÓPICA NO HOSPITAL MILITAR CENTRAL NO PERÍODO DE JANEIRO DE 2011 A JUNHO DE 2012.}

\section{Resumo}

Objetivos: Descrever as complicações e fatores de risco que ocorreram em pacientes submetidos à colangiopancreatografia retrógrada endoscópica (CPRE), no Hospital Militar Central no período de janeiro de 2011 a junho de 2012.

Métodos: Estudo retrospectivo descritivo. Revisão de prontuários médicos. Uma coorte de 96 pacientes submetidos a CPRE no Hospital Militar, enfatizando as características dos mesmos, indicação de CPRE e complicações apresentadas após este procedimento foi descrito. Realizou-se uma revisão da historia clinica dos pacientes com os critérios de inclusão, neles foi aplicado um questionário a fim de identificar quais as complicações ocorridas no período de tempo escolhido $e$ os fatores de risco que podem estar envolvidos. Com o teste do qui-quadrado foram avaliados a associação entre fatores de risco e complicações. 
Resultados: Foram encontrados que 20,8\% dos pacientes submetidos à CPRE apresentou uma complicação, a pancreatite aguda foi a mais comum com 11,5\%\%, seguido de infecção e sangramento gastrointestinal. A mortalidade foi de 2,1\%. A CPRE tinha indicação e foi realizada por pessoal treinado, do ponto de vista técnico. Não houve associação estatística entre os fatores de risco relacionados ao paciente ou técnica.

Conclusão: As complicações encontradas nos pacientes correspondem aos descritos na literatura, tanto no tipo e freqüência. Nenhuma associação estatisticamente significativa entre a ocorrência de complicações e os fatores de risco relacionados ao paciente ou a arte foi encontrado.

Palavras-chave: colangiopancreatografia endoscópica retrógrada, complicações, pancreatite, icterícia, perfuração intestinal.

\section{Introducción:}

La colangiopancreatografía retrograda endoscópica (CPRE), es hoy día, la principal técnica endoscópica terapéutica en el manejo de la enfermedad biliopancreática $(1,3,4)$, la cual podría incluir precorte, esfinterotomía biliopancreática, canulación biliopancreática, esfinteroplastia con balón, colocación de endoprótesis, dilatación de estenosis, litotripsia y toma de biopsia. Es la técnica más difícil de aprender y de realizar, por lo tanto a pesar de los adelantos tecnológicos, es el procedimiento con mayor morbimortalidad de la endoscopia digestiva. Las complicaciones de la CPRE incluyen pancreatitis aguda, colangitis, colecistitis, hemorragia postesfinterotomía, perforación retroperitoneal y la muerte $(2,3,9,14)$. La incidencia global de las complicaciones relacionadas con la CPRE varía dependiendo de la serie, entre $4-16 \%$, con una mortalidad de $0-1.5 \%(5,6,7)$. Se ha definido tres grupos de factores de riesgo de complicaciones típicas de la CPRE: relacionados con el paciente, con el endoscopista y con la técnica $(10,11,12,13)$; siendo la selección del paciente uno de los aspectos más importantes según la probabilidad de coledocolitiasis, de tal manera que aquellos con predictores muy fuertes de coledocolitiasis tendrían indicación excelente de CPRE (Tabla 1).

El propósito del presente estudio es describir las complicaciones que presentaron los pacientes en el Hospital Militar Central, debidas a la CPRE y los posibles factores asociados al desarrollo de las mismas.

\section{Materiales y métodos:}

Estudio descriptivo retrospectivo de pacientes sometidos a colangiopancreatografía retrograda endoscópica en el periodo de Enero de 2011 a Junio de 2012. La población accesible fueron adultos mayores de 18 años y la población elegible aquella que completó todos los datos en la revisión de la historia clínica y hayan sido sometidos a este procedimiento en el periodo escogido en nuestro estudio. $\mathrm{Se}$ excluyeron aquellos paciente cuya historia clínica no disponía de los datos completos que se recogían en la encuesta aplicada a las mismas. Se diseñó una encuesta para aplicar a las historias clinicas, que evaluaba los factores de riesgo descritos en la literatura así como las posibles complicaciones de la CPRE. Se revisaron las historias clínicas en el archivo de estadística, previa autorización por comité de ética del Hospital Militar Central; de igual manera, la historia clínica electrónica de la población seleccionada y los registros de CPRE del servicio de Gastroenterología del Hospital Militar Central. Se diligencio completamente el formato de recolección de datos con cada historia clínica. La historia clínica se revisó personalmente por los autores. Se realizó un análisis univariado utilizando medidas

Tabla 1. Riesgo de coledocolitiasis en pacientes con colelitiasis sintomatica basado en predictores clinicos (15)

\section{Predictores de coledocolitiasis}

\section{Muy fuertes}

- Lito en conducto bibiar comun (CBC) en ecografia transabdominal

- Clinica de colangitis ascendente

- Bilirrubinas totales $>4 \mathrm{mg} / \mathrm{dL}$

\section{Fuertes}

- $\mathrm{CBC}$ dilatado en ecografia ( $>6 \mathrm{~mm}$ con vesicula in situ) - Bilirrubinas totales entre 1.8 y $4 \mathrm{mg} / \mathrm{dL}$

\section{Moderados}

- Otras pruebas de bioquimica hepatica anormales diferentes de bilirrubinas - Edad > 55 años

- Pancreatitis de probable etiologia colelitiasica

\section{Interpretacion}

- Cualquier predictor muy fuerte: probabilidad alta

- Dos predictores fuertes: probabilidad alta

- Sin predictores: probabilidad baja

- Todos los otros pacientes: probabilidad intermedia. 
de tendencia central y de dispersión de tal manera que las variables cualitativas fueron expresadas con frecuencias absolutas y porcentajes y las variables cuantitativas con promedios y desviaciones estándar, medianas y cuartiles cuando aplicaba (variables sin distribución normal). Posteriormente se efectuó un análisis bivariado utilizando la prueba de Chi cuadrado y el test exacto de Fisher (cuando alguno de los conteos era menor o igual a 5) con el fin de identificar diferencias significativas en el comportamiento de los factores de riesgo entre los pacientes que sufrieron complicaciones y aquellos que no lo hicieron. El anterior análisis fue realizado con SPSS versión 1.11 .

\section{Resultados}

Se analizaron un total de 96 pacientes llevados a colangiopancreatografía retrógrada endoscópica (CPRE) en el Hospital Militar Central durante el periodo comprendido entre Enero de 2011 y Junio de 2012. El 3,1\% de dichos pacientes provenían de una atención ambulatoria $(n=3)$, $77,1 \%$ de una atención intrahospitalaria $(\mathrm{n}=74)$ y $19,8 \%$ del servicio de Urgencias ( $\mathrm{n}=19$ ) (Figura 1).

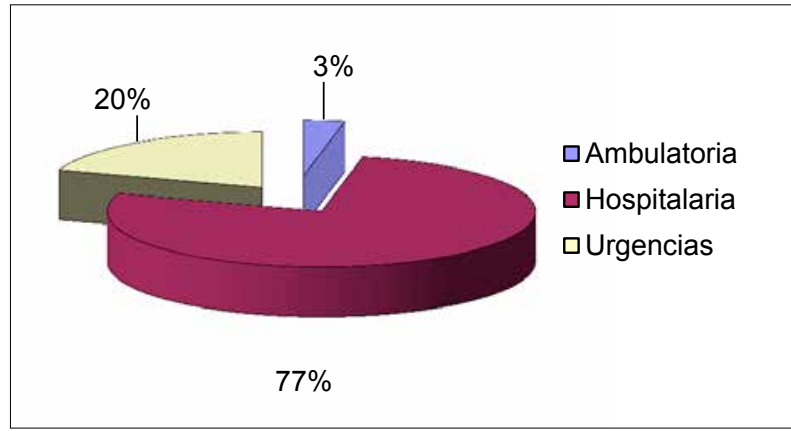

Figura 1. Distribución de los pacientes de acuerdo a su procedencia hospitalaria

\section{Características demográficas}

Un poco más de la mitad de los casos pertenecían al género masculino $(59,4 \%, n=57)$. La edad promedio observada fue 55,48 años con una desviación estándar de 18,77 años, encontrando pacientes con edades entre 18 y 68 años (Tabla 2).

El tiempo de estancia de dichos pacientes fue en promedio de $12.79 \pm 14,19$ días, evidenciando que en el $50 \%$ de los pacientes la duración de su hospitalización fue mayor de 9 días (Tabla 2).
Tabla 2. Características demográficas de los pacientes sometidos a CPRE en el Hospital Militar

\begin{tabular}{|l|l|}
\hline \multicolumn{1}{|c|}{ Característica } & \multicolumn{1}{c|}{ n (\%) } \\
\hline $\begin{array}{l}\text { Género } \\
\text { Masculino }\end{array}$ & $57(59,4 \%)$ \\
Femenino & $39(40,6 \%$ \\
\hline Edad & $55.48 \pm 18.77$ \\
Promedio \pm ds & 57 \\
Mediana & $18-88$ \\
Mínimo - Màximo & \\
\hline Estancia (días) & $12.79 \pm 14,19$ \\
Promedio \pm ds & 9 \\
Mediana & $2-104$ \\
Mínimo - Màximo & \\
\hline Peso (Kg) & $67,94 \pm 12,21$ \\
Promedio \pm ds & 68 \\
Mediana & $33-97$ \\
Mínimo - Màximo & \\
\hline Talla (cm) & $1,64 \pm 0,07$ \\
Promedio \pm ds & 1,64 \\
Mediana & $1,41-178$ \\
Mínimo - Màximo & \\
\hline IMC & $25,27 \pm 4,8$ \\
Promedio \pm ds & 24,2 \\
Mediana & $13,7-46,11$ \\
Mínimo - Màximo &
\end{tabular}

\section{Antecedentes}

La mayor parte de los pacientes a quienes se les realizó el procedimiento tenían antecedente de ictericia $(87,5 \%$, $\mathrm{n}=84$ ). Dentro de las comorbilidades la más frecuente fue la Hipertensión arterial presente en 28 pacientes (29,2\%), seguida por la Enfermedad Pulmonar Obstructiva Crónica encontrada en 9 casos $(9,4 \%)$ y por el tabaquismo en 8 casos $(8,3 \%)$ (Tabla 3$)$

Con relación a los antecedentes farmacológicos relevantes se evidenció que $17,7 \%$ se encontraban manejados con AINES ( $n=17), 2,1 \%$ con Warfarina $(n=2)$ y $5,2 \%$ con $\mathrm{He}^{-}$ parina $(\mathrm{n}=5)$ (Tabla 3$)$.

Por otra parte, sólo se encontró un paciente con antecedente de cirugía gástrica previa (1\%).

\section{Laboratorios}

Con respecto a las pruebas de coagulación se evidenció que los pacientes analizados tenían un promedio de tiempo de protrombina de 11,47 segundos con una desviación estándar de 1,49 segundos y un tiempo parcial de trombo- 
Tabla 3. Antecedentes de los pacientes sometidos a CPRE en el Hospital Militar

\begin{tabular}{|l|r|}
\hline \multicolumn{1}{|c|}{ Antecedente } & n (\%) \\
\hline Patológicos & \\
Ictericia & $84(87,5 \%)$ \\
Hipertensión arterial & $28(29,2 \%)$ \\
EPOC & $9(9,4 \%)$ \\
Tabaquismo & $8(8,3 \%)$ \\
Falla cardiaca & $4(4,2 \%)$ \\
Diabetes mellitas & $3(3,1 \%)$ \\
Inmunosupresión & 0 \\
\hline Farmacológicos & \\
AINES & $17(17,7 \%)$ \\
Warfarina & $2(2,1 \%)$ \\
Heparina & $5(5,2 \%)$ \\
Ninguno & $75(78,1 \%)$ \\
\hline Cirugía gástrica previa & $1(1 \%)$ \\
\hline
\end{tabular}

plastina promedio de 29,22 segundos con una desviación estándar de 4,44 segundos. El INR osciló entre 0,89 y 2,04 (Tabla 4).

El promedio de la hemoglobina disminuyó en las primeras 24 horas posteriores al procedimiento, pasando de 14,06 $\pm 1,84 \mathrm{mg} / \mathrm{dl} \mathrm{a} 11,95 \pm 7,28 \mathrm{mg} / \mathrm{dl}$. Sin embargo, dichos niveles volvieron a incrementarse a las 48 horas de la CPRE alcanzando un promedio de 13,95 5 5,02 mg/dl (Tabla 4).

Por otra parte, los niveles de amilasa (solo en pacientes que presentaron pancreatitis aguda) a las 24 horas de realizado el procedimiento oscilaron entre 329 y $3370 \mathrm{mg} /$ $\mathrm{dl}$, con un promedio de $1178,78 \pm 922,93$. El $50 \%$ de los pacientes tenían en dicho momento una amilasa por encima de $980 \mathrm{mg} / \mathrm{dl}$ (Tabla 4).

\section{Características de la Colangiografía retrógrada endoscópica (CPRE)}

En la totalidad de los casos estudiados la CPRE fue realizada por un endoscopista que había realizado más de 50 procedimientos (Tabla 5).

La sedación y analgesia fue realizada con Butil bromuro de hioscina en 83 pacientes (86,5\%), con Midazolam en 69 pacientes $(71,9 \%)$, con Propofol en 58 pacientes $(60,4 \%)$, con Meperidina en 48 pacientes $(n=50 \%)$ y con Fentanyl en 15 pacientes (15,6\%). Solo hubo un paciente que requirió anestesia general para la realización del procedimiento (Tabla 5).
Tabla 4. Comportamiento de los estudios paraclínicos entre los pacientes sometidos a CPRE en el Hospital Militar

\begin{tabular}{|c|c|}
\hline $\begin{array}{l}\text { Tiempo de protrombina }(\mathbf{s e g}) \\
\text { Promedio } \pm \mathrm{ds} \\
\text { Mediana } \\
\text { Mínimo - Màximo }\end{array}$ & $\begin{array}{c}11,47 \pm 1,49 \\
11,1 \\
9,3-16,6\end{array}$ \\
\hline $\begin{array}{l}\text { Tiempo parcial de tromboplastina } \\
\text { (seg) } \\
\text { Promedio } \pm \text { ds } \\
\text { Mediana } \\
\text { Mínimo - Màximo }\end{array}$ & $\begin{array}{c}29,22 \pm 4,44 \\
27,85 \\
18,6-44,7\end{array}$ \\
\hline $\begin{array}{l}\text { INR } \\
\text { Promedio } \pm \text { ds } \\
\text { Mediana } \\
\text { Mínimo - Màximo }\end{array}$ & $\begin{array}{l}1.15 \pm 0.19 \\
1.10 \\
0.89-2.04\end{array}$ \\
\hline $\begin{array}{l}\text { Hemoglobina previa }(\mathbf{m g} / \mathbf{d l}) \\
\text { Promedio } \pm \mathrm{ds} \\
\text { Mediana } \\
\text { Mínimo - Màximo }\end{array}$ & $\begin{array}{c}14,06 \pm 1,84 \\
14,05 \\
9,8-18,4\end{array}$ \\
\hline $\begin{array}{l}\text { Hemoglobina a las } 24 \mathbf{h}(\mathbf{m g} / \mathbf{d l}) \\
\text { Promedio } \pm \text { ds } \\
\text { Mediana } \\
\text { Mínimo - Màximo }\end{array}$ & $\begin{array}{c}11,95 \pm 7,28 \\
11,95 \\
6,8-17,1\end{array}$ \\
\hline $\begin{array}{l}\text { Hemoglobina a las } \mathbf{4 8 h}(\mathbf{m g} / \mathbf{d l}) \\
\text { Promedio } \pm \text { ds } \\
\text { Mediana } \\
\text { Mínimo - Màximo }\end{array}$ & $\begin{array}{c}13,95 \pm 5,02 \\
13,95 \\
10,4-17,5\end{array}$ \\
\hline $\begin{array}{l}\text { Amilasa a las } 24 h(\mathbf{h g} / \mathbf{d l}) * \\
\text { Promedio } \pm \mathrm{ds} \\
\text { Mediana } \\
\text { Mínimo - Màximo }\end{array}$ & $\begin{array}{c}1178,78 \pm 922,93 \\
980 \\
329-3370\end{array}$ \\
\hline
\end{tabular}

* Solo medida en pacientes que presentaron sintomas sugestivos de pancreatitis post $\mathrm{CPRE}$.

En un poco más de la mitad de los pacientes $(55,2 \%$, $\mathrm{n}=53$ ) se realizaron menos de 4 intentos para canular la papila y en 6 casos (6,3\%) se logró la canulación del conducto pancreático (Tabla 5).

Se encontraron 14 pacientes con divertículo yuxtapapilar $(14,6 \%)$ y 13 pacientes con disfunción del esfínter de Oddi $(13,5 \%)$. En la mayor parte de los casos el diámetro del colédoco se observó mayor o igual a $8 \mathrm{~mm}(66,7 \%, \mathrm{n}=64)$ (Tabla 5).

Dentro de los procedimientos realizados durante la CPRE se evidenció que a 37 pacientes se les hizo precorte $(38,5 \%)$, a 23 pacientes se les colocó una endoprótesis $(24 \%)$, a 1 paciente se le realizó esfinterotomía pancreática (1\%) y a 1 paciente se le hizo esfinteroplastia con balón (1\%) (Tabla 5).

Durante la colangiografía los pacientes tuvieron una tensión arterial sistólica promedio de $121,86 \mathrm{mmHg}$, una tensión arterial diastólica promedio de 74,68 $\mathrm{mmHg}$ y una tensión arterial media de $91,05 \mathrm{mmHg}$. En cuanto a 
Tabla 5. Características de la CPRE

\begin{tabular}{|l|c|}
\hline \multicolumn{1}{|c|}{ Característica } & n (\%) \\
\hline Sedoanalgesia & \\
Butil bromuro de hioscina & $83(86,5 \%)$ \\
Midazolam & $69(71,9 \%)$ \\
Propofol & $58(60,4 \%)$ \\
Meperidina & $48(50 \%)$ \\
Fentanyl & $15(15,6 \%)$ \\
\hline Anestesia general & $1(1 \%)$ \\
\hline Intentos de canular la papila & \\
< 4 intentos & $53(55,2 \%)$ \\
\hline intentos & $52(43,8 \%)$ \\
Sin dato & $1(1 \%)$ \\
\hline Canulación del conducto pancreàtico & \\
Si & $6(6,3 \%)$ \\
No & $90(93,8 \%)$ \\
\hline Divertículo yuxtapapilar & \\
Si & $14(14,6 \%)$ \\
No & $82(85,4 \%)$ \\
\hline Disfunción esfínter de Oddi & $13(13,5 \%)$ \\
\hline Colédoco & \\
< 8 mm & $32(33,3 \%)$ \\
8 mm & $64(66,7 \%)$ \\
\hline Precorte & $37(38,5 \%)$ \\
\hline Endoprótesis & $23(24 \%)$ \\
\hline Esfinterotomía pancreática & $1(1 \%)$ \\
\hline Esfinteroplastia con balón & $1(1 \%)$ \\
\hline Dilatación & 0 \\
\hline Indicación & $1(1 \%)$ \\
Excelente & $24(25 \%)$ \\
Regular & $1(1 \%)$ \\
Mala & \\
Sin dato & \\
\hline
\end{tabular}

la saturación arterial de Oxígeno el promedio observado fue $93,02 \%$

El porcentaje de pacientes con una excelente indicación para la realización de la colangiografía fue 72,9\% ( $\mathrm{n}=70)$, encontrando un solo caso con mala indicación (1\%).

Durante el procedimiento sólo hubo 2 pacientes que requirieron transfusión de GRE. En ambos casos el total de unidades transfundidas fue 3 .

\section{Complicaciones}

Del total de la población analizada se presentaron complicaciones en el 20,8\% ( $n=20)$. Dentro de las complicaciones hubo 11 casos con pancreatitis (11,5\%), 4 pacientes con infección $(4,2 \%), 3$ pacientes con sangrado digestivo $(3,1 \%), 3$ pacientes con hemorragia $(3,1 \%), 2$ pacientes con perforación $(2,1 \%)$ en ambos casos del duodeno, 1 paciente con neumoperitoneo (1\%) y 1 paciente con migración del stent (1\%) (Figura 2 ).

Adicionalmente, 16 pacientes cursaron con desaturación $(16,7 \%)$ y 13 pacientes con dolor abdominal a las 24 horas del procedimiento $(13,5 \%)$.

El porcentaje de mortalidad observado fue 2,1\%, representando dos casos, ambos del sexo masculino, de 43 y 84 años de edad; el primer caso, correspondía a un paciente que tenía indicación regular de CPRE, la complicación presentada fue perforación intestinal, la cual acontecio a los 7 dias del procedimiento. El segundo caso fue un paciente con indicación excelente de CPRE, con complicación dada por perforación intestinal que ocurrió a los 6 días, y tenía

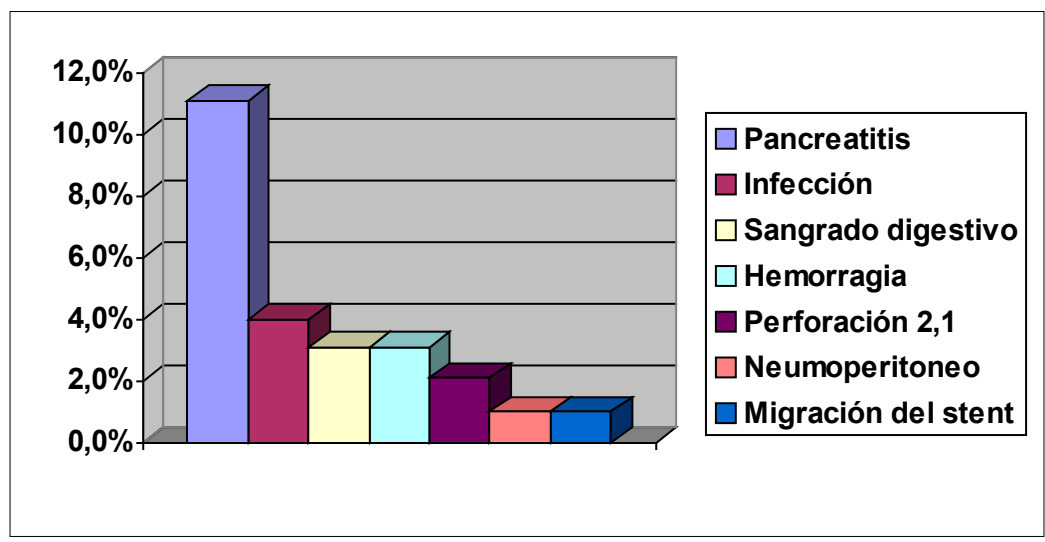

Figura 2. Complicaciones presentadas entre los pacientes sometidos a CPRE en el Hospital Militar. 
como factores de riesgo la presencia de diverticulo yuxtapapilar y ausencia de ictericia.

No se encontró ninguna asociación estadísticamente significativa entre la ocurrencia de complicaciones y aquellos factores de riesgo relacionados con el paciente, descritos en la literatura. Entre los pacientes que se complicaron como en aquellos que no se complicaron hubo una mayor proporción de casos pertenecientes al género masculino, así como una mayor proporción de casos con ictericia. De igual forma, en ambos grupos de pacientes hubo mayor porcentaje de casos con diámetro del colédoco mayor o igual a $8 \mathrm{~mm}$ así como una proporción similar de casos con una indicación regular para la realización de la colangiografía (Tabla 6).

Por otra parte, tampoco se encontraron asociaciones significativas entre la ocurrencia de complicaciones y aquellos factores de riesgo relacionados con la técnica. El porcentaje de pacientes con más de 4 intentos fue muy similar en ambos grupos (50\% entre los complicados y 42,1\% entre los no complicados). Así mismo la proporción de casos a quienes se les realizó precorte así como de aquellos a quienes se les colocó endoprótesis también fue muy parecido (Tabla 7).

\section{Discusión}

Las complicaciones más frecuentes en el Hospital Militar Central en este periodo fueron pancreatitis, infección y sangrado digestivo, lo cual es consistente con lo descrito por la literatura en series de casos descriptivos acerca de los eventos adversos de este procedimiento $(2,8)$. No obstante, es de resaltar que el porcentaje encontrado de pancreatitis es ligeramente mayor al mencionado en los estudios descriptivos que encontramos en nuestra revisión bibliográfica (1, 2, 3). Así mismo, la mortalidad encontrada en nuestros pacientes fue algo mayor $(5,6,7)$; esto probablemente obedezca a la relativa facilidad para demostrar el diagnóstico de pancreatitis como complicación de CPRE o al subregistro de las otras complicaciones mencionadas en nuestros resultados. Si bien es cierto la mayoría de las CPRE tenían indicación excelente, un porcentaje no despreciable (25\%), correspondia a indicación regular, lo cual podría estar en relación con la ausencia de los métodos auxiliares diagnósticos utilizados en estos casos (colangiorresonancia y la ecoendoscopia) así como la experiencia local requerida para su realización en nuestra institución. De tal manera que en aras de evitar el deterioro clínico de nuestros pacientes se realiza la CPRE, lo cual también está usustentado por las guías de manejo de coledocolitiasis
(15). Contrariamente a lo esperado, en nuestro estudio el sexo masculino fue el más afectado con complicaciones.

Todas las CPRE fueron realizadas por personal idóneo que cumplía con los requerimientos técnicos mínimos que garantizan la pericia para la realización de dicho procedimiento. La mayoría de las CPRE estaban adecuadamente indicadas. No podemos dejar de mencionar que el Hospital Militar Central es un centro de entrenamiento para residentes, y aun así la frecuencia de complicaciones fue similar a lo reportado en la literatura. Sin embargo tenemos como limitación en nuestro estudio que el periodo de tiempo seleccionado es corto, lo que podría subestimar el porcentaje de CPRE encontrado con indicación regular o mala, por lo tanto proponemos como mecanismo de retroalimentación y mejoramiento, la ampliación de esta línea de estudio tanto de forma retrospectiva, como el seguimiento prospectivo de los casos venideros, de tal manera que se puedan identificar las causas de una inadecuada selección de los pacientes que serian sometidos a CPRE, que es en definitiva el factor de riesgo más importante para que sucedan complicaciones $(3,11,13)$. No encontramos asociación estadísticamente significativa entre las complicaciones y los factores de riesgo relacionados con el paciente y la técnica.

Conflicto de Intereses: no reportamos ninguno.

\section{Referencias}

1. Freeman ML. Complications of endoscopic retrograde cholangiopancreatography: avoidance and management. Division of Gastroenterology, Hepatology and Nutrition, University of Minnesota. Gastrointest Endosc Clin N Am. 2012 Jul;22(3):567-86

2. F.J. Gallego Rojo, F. Gallardo Sánchez. Complicaciones de la colangiopancreatrografía retrógrada endoscópica. Identificación, prevención y manejo. Rev. Andal. Patol. Dig. 2010;33(1): 22-36

3. Penaloza-Ramirez, A.; Leal-Buitrago, C. y Rodriguez-Hernandez, A. Adverse events of ERCP at San José Hospital of Bogotá (Colombia). Rev. Esp. Enferm. Dig. 2009;101(12): 837843

4. Huibregtese K. Complications of endoscopic sphincterotomy and their prevention. N Engl J Med 1996; 335: 961-3.

5. Freeman ML, Nelson DB, Sherman S, Haber GB, Herman ME, Dorsher PJ, et al: Complications of endoscopic biliary sphincterotomy. N Engl J Med 1996; 335:909-18.

6. Loperfido S, Angelini G, Benedetti G, Chilovi F, Costan F, De Berardinis F, et al: Major early complications from diagnostic and therapeutic ERCP: a prospective multicenter study. Gastrointest Endosc 1998;48:1-10.

7. Masci E, Toti G, Mariani A, Curioni S, Lomazzi A, Dinelli M, et al: Complications of diagnostic and therapeutic ERCP: a pro- 
spective multicenter study. Am J Gastroenterol 2001;96:417423.

8. Cotton PB, Lehman G, Vennes J, Geenen JE, Russell RC, Meyers WC et al. Endoscopic sphincterotomy complications and their management: an attempt at consensus. Gastrointest Endosc 1991; 37: 383-93.

9. García-Cano J. Severe cardiogenic pulmonary edema precipitated by a therapeutic ERCP. Endoscopy 2004;36:567-8.

10. Cotton PB, Leung J. Advanced digestive Endoscopy: ERCP, Blackwell Publishing, editor. Massachusetts, 2006:339-403.

11. Christensen M, Matzen P, Schulze S, Rosenberg J.: Complications of ERCP: a prospective study. Gastrointest Endosc 2004; 60:721-31
12. Freeman ML. Adverse outcomes of ERCP. Gastrointest Endosc 2002; 56:S273-82.

13. Leung JW, Chung SC, Sung JJ, Banez VP, Li AK. Urgent endoscopic drainage for acute suppurative cholangitis. Lancet 1989; 1 (8650): 1307-9.

14. Veldkamp MC, Rauws EA, Dijkgraaf MG, Fockens P, Bruno MJ. Iatrogenic ampullary stenosis: history, endoscopic management, and outcome in a series of 49 patients. Gastrointest Endosc. 2007; 66(4):708-16.

15. The role of endoscopy in the evaluation of suspected choledocholithiasis. Communication from the ASGE Standards of Practice Commite.Gastrointest Endosc. 2010; 71 (1). 